\title{
Comparison of Organic and Conventional Crops at the Neely-Kinyon LTAR Site, 2001
}

\author{
Kathleen Delate, assistant professor \\ Departments of Horticulture and Agronomy \\ Cynthia Cambardella, soil scientist \\ USDA National Soil Tilth Lab \\ Bob Burcham, farm supervisor \\ Neely-Kinyon Research and \\ Demonstration Farm \\ Heather Friedrich and Noreen Wantate, \\ research associates \\ Departments of Horticulture and Agronomy
}

\section{Introduction}

Sales of organic products reached $\$ 8$ billion industry in the United States in 2001, continuing a $20 \%$ annual growth rate. In Iowa alone, reported acreage for all organic crops increased from 13,000 in 1995 to 100,000 in 2000. With support from the Leopold Center for Sustainable Agriculture, Long-Term Agroecological Research (LTAR) sites were established throughout Iowa in 1998 to examine the agronomic, environmental, and economic effects of organic practices over the long term. Neely-Kinyon organic LTAR plots became certified organic in 2000. Results from 2001 represent crop yields after one full rotation.

\section{Materials and Methods}

Treatments, replicated four times, in the LTAR site included conventional corn-soybean, organic corn-soybean-oats/alfalfa, organic corn-soybean-oats/alfalfa-alfalfa, and soybean-wheat/crimson clover. The soybean-wheat/crimson clover rotation replaced the soybean/winter rye rotation that was planted from 1998-2000. New certification regulations introduced by the State of Iowa Organic program in 2000 disallowed the soybean-rye rotation because the rye was not grown for a full season.

Variety selection and planting methods were as follows:
October 16, 2000, following harvest of the organic corn plots, winter rye was no-till drilled at a rate of 1 bushel/acre.

October 24, 2000, Arapahoe winter wheat was planted at $90 \mathrm{lb} / \mathrm{acre}$; with crimson clover frostseeded into the wheat plots on March 27, 2001, at $10 \mathrm{lb} / \mathrm{acre}$.

April 16, 2001, organic corn plots were fertilized with compost at 12 tons/acre;. May 16, conventional corn plots were fertilized with $28 \%$ urea at $125 \mathrm{lb} /$ acre N. On May 18, 2001, Pioneer '34W67' corn was planted 1.75 inches deep as untreated seed at 32,000 seeds/acre in the organic plots and as treated seed at 30,200 seeds/acre in conventional plots.

Also on April 16, 2001, oat plots were fertilized with compost at 5 tons/acre. On April 18, Blaze oats were underseeded with Pioneer 53H81 leafhopper-tolerant alfalfa at a depth of 0.5 inches, at 3.2 bushels/acre and $20 \mathrm{lb} / \mathrm{acre}$, respectively.

May 29, 2001, Northrup King 2412 soybeans were planted two inches deep in organic and conventional plots at 170,000 seeds/acre. Soybeans were replanted due to insufficient stands on June 16, 2001.

The following pest management applications were made in the 2001 season:

May 16, 2001, conventional corn plots were sprayed with Harness ${ }^{\circledR}(2.5 \mathrm{pt} / \mathrm{acre})$ and Atrazine $^{\circledR}$ (1pt/acre) then, June 20 with Buctril ${ }^{\circledR}$ (1pt/acre), Accent ( 0.67 oz/acre), AMS (2 lbs/acre), and NIS ${ }^{\circledR}$ (0.4 pt/acre). Organic corn plots were harrowed on June 8, 2001, and cultivated June 11, June 13, and June 26. June 6, 2001, conventional soybean plots were sprayed with Prowl ${ }^{\circledR}$ at (2.2 pts/acre), then, July 10 , with Manifest G (2 pts/acre), Select ${ }^{\circledR}$ 
(12oz/acre), $\operatorname{AMS}^{\circledR}$ (2 lbs/acre), and NIS ${ }^{\circledR}(0.3$ pts/acre). Soybean plots were field cultivated before replanting on June 16. Organic soybeans were rotary hoed on June 25 and cultivated on July 6,13 , and 30 .

Stand counts were taken in corn plots on June 8 and in soybean plots on July 5. Using square meter quadrants at three randomly selected areas within a plot, weed counts were taken in corn plots June 8 and July 5, and in soybean plots June 29 and July 31. Corn borer populations were monitored July 12 . Soybean plots were sampled for bean leaf beetles July 19 and 26, Aug. 10, and Sept. 13. Soybean cyst nematode sampling was conducted September 19 by collecting one pint of soil from each soybean plot to a 6-inch depth.

Samples were analyzed for SCN populations at the ISU Plant Disease Clinic. Soil in corn plots was sampled on June 28 and analyzed for late spring nitrate by the USDA-ARS National Soil Tilth Laboratory, Ames, Iowa. Stalk nitrate sampling occurred October 4, 2001. All tissue samples were analyzed in the USDA-ARS National Soil Tilth Laboratory.

Half of each wheat/crimson clover plot was mowed with a disc mower and baled on June 25, 2001. Alfalfa was mowed and baled July 12 and August 8. Oat and wheat plots were combined July 25. Corn and soybeans were harvested on October 27. Samples were collected from each plot for grain analysis. Corn grain quality analyses for protein, oil, and starch was conducted at the ISU Grain Quality Laboratory.

\section{Results and Discussion}

Organic corn and soybean yields were significantly greater than conventional yields in 2001 , with greatest yields following two years of alfalfa. Organic corn yields averaged 130 bushels/acre, and conventional 112 bushels/acre. Conventional soybean yields averaged 40 bushels/acre, while organic soybeans yielded 45 bushels/acre. Wheat yields averaged 39 bushels/acre, and oat yields averaged 82 bushels/acre. Second-year alfalfa plots averaged 3.4 tons/acre.

Corn and soybean populations were similar among treatments after 3 weeks. Early grass weed populations in organic corn plots were greater in plots following two years of alfalfa, but broadleaf weeds were greater in $\mathrm{C}-\mathrm{SB}-\mathrm{O} / \mathrm{A}$ plots. Weed populations were less in soybean plots, with similar levels in all organic treatments on July 31. Corn borer populations were below economic thresholds and similar among treatments. Bean leaf beetle populations also were similar between conventional and organic plots. Stalk nitrate levels were highest in conventional corn $(5,543 \mathrm{ppm})$, although not significantly greater than organic corn $(3,048)$. Significantly higher levels of protein were found in conventional corn (9.0\% vs. $8.5 \%)$. Similar levels of protein $(33 \%)$ were found among soybean plots, but greater levels of carbohydrates were found in organic soybeans. Soil quality analysis currently is underway. Organic crops were sold to Heartland Organic Marketing Cooperative at a $200 \%$ premium over conventional 2001 prices. 
Table 1. Organic and conventional grain crop yields at Neely-Kinyon, Greenfield, IA, 2001.

\begin{tabular}{lllll} 
Treatment & $\begin{array}{l}\text { Corn yield } \\
\text { bu/ac }\end{array}$ & $\begin{array}{l}\text { Soybean yield } \\
\text { bu/acre }\end{array}$ & $\begin{array}{l}\text { Oat yield } \\
\text { bu/acre }\end{array}$ & $\begin{array}{l}\text { Wheat yield } \\
\text { bu/acre }\end{array}$ \\
Conv. C-SB & $112.41 \pm 3.46$ & $39.93 \pm 0.60$ & N/A & N/A \\
Org. C-SB-O/A & $127.90 \pm 3.50$ & $42.82 \pm .058$ & $81.10 \pm 1.19$ & N/A \\
Org. C-SB-O/A-A & $131.43 \pm 4.65$ & $46.92 \pm 0.70$ & $82.80 \pm 3.08$ & N/A \\
Org. SB-W & N/A & N/A & N/A & $38.19 \pm 3.64$ \\
LSD $(0.05)$ & 11.51 & 1.85 & NSD & N/A \\
\hline
\end{tabular}

Table 2. Corn stands and weed populations in corn plots at Neely-Kinyon, Greenfield, IA, 2001.

\begin{tabular}{|c|c|c|c|c|c|}
\hline \multirow[t]{2}{*}{ Treatment } & \multirow[t]{2}{*}{$\begin{array}{l}\text { Corn stands } \\
\text { plants/acre }\end{array}$} & \multicolumn{2}{|c|}{$\begin{array}{c}\text { Corn weeds/m² } \\
\text { June 8, 2001 }\end{array}$} & \multicolumn{2}{|c|}{$\begin{array}{c}\text { Corn weeds/m² } \\
\text { July 5, 2001 }\end{array}$} \\
\hline & & Grasses & Broadleaves & Grasses & Broadleaves \\
\hline $\mathrm{C}-\mathrm{SB}$ & $28,750 \pm 1533$ & $0.00 \pm 0.00$ & $2.08 \pm 0.95$ & $1.83 \pm 0.51$ & $3.42 \pm 3.15$ \\
\hline $\mathrm{C}-\mathrm{SB}-\mathrm{O} / \mathrm{A}$ & $29,080 \pm 633$ & $10.00 \pm 3.28$ & $33.42 \pm 7.9$ & $5.50 \pm 2.38$ & $0.75 \pm 0.58$ \\
\hline $\mathrm{C}-\mathrm{SB}-\mathrm{O} / \mathrm{A}-\mathrm{A}$ & $30,170 \pm 985$ & $135.42 \pm 14.57$ & $21.25 \pm 5.9$ & $4.75 \pm 1.61$ & $13.83 \pm 2.75$ \\
\hline LSD (0.05) & NSD & 24.81 & 16.13 & NSD & 7.00 \\
\hline
\end{tabular}

Table 3. Soybean stands and weed populations in soybean plots at Neely-Kinyon, Greenfield, IA, 2001.

\begin{tabular}{|c|c|c|c|c|c|}
\hline \multirow[t]{2}{*}{ Treatment } & \multirow[t]{2}{*}{$\begin{array}{l}\text { Soybean stands } \\
\text { plants/acre }\end{array}$} & \multicolumn{2}{|c|}{$\begin{array}{c}\text { Soybean weeds/ } \mathbf{m}^{2} \\
\text { June } 29,2001\end{array}$} & \multicolumn{2}{|c|}{$\begin{array}{c}\text { Soybean weeds/ } \text { m }^{2} \\
\text { July } 31,2001\end{array}$} \\
\hline & & Grasses & Broadleaves & Grasses & Broadleaves \\
\hline $\mathrm{C}-\mathrm{SB}$ & $124,330 \pm 3424$ & $0.17 \pm 0.11$ & $1.67 \pm 0.64$ & $0.00 \pm 0.00$ & $2.58 \pm 0.61$ \\
\hline $\mathrm{C}-\mathrm{SB}-\mathrm{O} / \mathrm{A}$ & $112,080 \pm 6751$ & $2.58 \pm 0.57$ & $4.42 \pm 0.87$ & $0.67 \pm 0.26$ & $1.00 \pm 0.49$ \\
\hline $\mathrm{C}-\mathrm{SB}-\mathrm{O} / \mathrm{A}-\mathrm{A}$ & $121,333 \pm 3970$ & $6.08 \pm 1.55$ & $5.58 \pm 1.32$ & $1.00 \pm 0.60$ & $1.67 \pm 0.72$ \\
\hline LSD (0.05) & NSD & 2.74 & 2.84 & NSD & NSD \\
\hline
\end{tabular}

Table 4. Pest populations and stained soybeans from soybean plots at Neely-Kinyon, Greenfield IA, 2001.

\begin{tabular}{lllll} 
Treatment & $\begin{array}{l}\text { Corn borer } \\
\text { damage }\end{array}$ & \multicolumn{1}{c}{ Bean leaf beetles } & $\begin{array}{c}\text { Stained soybean } \\
(\boldsymbol{\%})\end{array}$ & $\begin{array}{l}\text { Soybean cyst nematode } \\
(\mathbf{e g g s} \text { per 100 cc) }\end{array}$ \\
& $0.22 \pm 0.15$ & $2.75 \pm 0.95$ & $8.16 \pm 0.84$ & $387.50 \pm 283.12$ \\
C-SB & $0.18 \pm 0.18$ & $1.75 \pm 0.63$ & $11.93 \pm 1.89$ & $62.50 \pm 62.5$ \\
C-SB-O/A & $1.75 \pm 0.48$ & $17.70 \pm 2.02$ & $150.00 \pm 150.0$ \\
C-SB-O/A-A & $0.17 \pm 0.11$ & NSD & 5.33 & NSD \\
LSD $(0.05)$ & NSD & &
\end{tabular}

Table 5. Corn stalk nitrate and grain quality at Neely-Kinyon, Greenfield, IA, 2001.

\begin{tabular}{|c|c|c|c|c|c|c|}
\hline \multirow[t]{2}{*}{ Treatment } & \multirow{2}{*}{$\begin{array}{l}\text { Stalk nitrate } \\
\quad(\mathbf{p p m})\end{array}$} & \multicolumn{5}{|c|}{2001 Corn grain quality (\%) } \\
\hline & & Density & Starch & Oil & Protein & Moisture \\
\hline $\mathrm{C}-\mathrm{SB}$ & $5542.5 \pm 1136.2$ & $1.31 \pm 0.002$ & $59.68 \pm 0.11$ & $3.63 \pm 0.05$ & $9.00 \pm 0.07$ & $18.65 \pm 0.26$ \\
\hline $\mathrm{C}-\mathrm{SB}-\mathrm{O} / \mathrm{A}$ & $2664.5 \pm 1213.4$ & $1.31 \pm 0.002$ & $60.10 \pm 0.14$ & $3.73 \pm 0.08$ & $8.43 \pm 0.08$ & $18.23 \pm 0.29$ \\
\hline $\mathrm{C}-\mathrm{SB}-\mathrm{O} / \mathrm{A}-\mathrm{A}$ & $3430.0 \pm 1109.3$ & $1.32 \pm 0.001$ & $59.93 \pm 0.09$ & $3.70 \pm 0.04$ & $8.60 \pm 0.12$ & $18.25 \pm 0.2$ \\
\hline $\operatorname{LSD}(0.05)$ & NSD & NSD & NSD & NSD & 0.296 & NSD \\
\hline
\end{tabular}

Table 6. Soybean grain quality at Neely-Kinyon, Greenfield, IA, 2001.

\begin{tabular}{lllllc}
\hline Treatment & & \multicolumn{2}{c}{ 2001 Soybean grain quality (\%) } \\
& Carbohydrates & Fiber & Oil & Protein & Moisture \\
C-SB & $24.00 \pm 0.16$ & $4.63 \pm 0.06$ & $20.60 \pm 0.49$ & $32.78 \pm 0.63$ & $12.50 \pm 0.11$ \\
C-SB-O/A & $24.08 \pm 0.05$ & $4.63 \pm 0.09$ & $20.70 \pm 0.09$ & $32.60 \pm 0.20$ & $12.50 \pm 0.06$ \\
C-SB-O/A-A & $23.50 \pm 0.08$ & $4.58 \pm 0.03$ & $20.05 \pm 0.1$ & $33.88 \pm 0.14$ & $12.43 \pm 0.18$ \\
LSD $(0.05)$ & 0.34 & NSD & NSD & NSD & NSD \\
\hline
\end{tabular}

